\title{
A COINCIDENCE THEOREM IN TOPOLOGICAL
}

\section{VECTOR SPACES}

\author{
OLGA HADŽI ́́
}

In this paper we prove a coincidence theorem in not necessarily locally convex topological vector spaces, which contains, as a special case, a coincidence theorem proved by Felix Browder. As an application, a result about the existence of maximal elements is obtained.

\section{Introduction}

In the recent time many papers are devoted to the fixed point theory in not necessarily locally convex topological vector spaces [3][13].

There are many important topological vector spaces which are not locally convex, as for example $L^{p}(0<p<1), S(0,1)$ (the space of all equivalence classes of measurable functions on $[0,1]) . H^{p}(0<p<1)$. So, it is of interest to find fixed point theorems for mappings defined on such spaces. The book [7] contains the most important results from the fixed point theory in topological vector spaces. In this paper we prove some generalizations of Proposition 2 and Theorem 1 from Browder's paper [1] to topological vector spaces which are not necessarily locally convex.

Received 31 July 1985.

Copyright Clearance Centre, Inc. Serial-fee code: 0004-9727/86 $\$ A 2.00+0.00$. 
First, we shall give some notations and definitions.

Let $E$ be a topological vector space and $M \subseteq E$. It is assumed that all topological spaces in this paper are Hausdorff.

By $2_{c o}^{M}$ we shall denote the family of all nonempty, convex subsets of $M$. Let $F$ be an another topological vector space, $C$ a nonempty subset of $E$ and $T$ a multivalued mapping from $C$ into $F$. The mapping $T$ is said to be upper semicontinuous if for each neighbourhood $V$ of zero in $F$ and each point $x_{0} \in C$, there exists a neighbourhood $U$ of zero in $E$ such that:

$$
T(x) \subseteq T\left(x_{0}\right)+V \text {, for all } x \in\left(x_{0}+U\right) \cap C \text {. }
$$

In [5] we introduced the following definition.

DEFINITION 1. Let $E$ be a topological vector space, $K \subseteq E$ and $U$ the fundomental system of neighbourhoods of zero in $E$. The set $K$ is said to be of Zima's type if for every $V \in U$ there exists $U \in U$ such that:

$$
\text { co }(U \cap(K-K)) \subseteq V \quad(\text { co is the convex huZl). }
$$

Remark. In [6] we proved the following result: If $K$ is a convex subset of zima's type of a Hausdorff topological vector space $E$ then:

$$
A \subseteq K, A \text { is precompact } \Rightarrow c 0 A \text { is precompact. }
$$

By (1) we proved in [6] a generalization of Sadovski's fixed point theorem.

Let us give an example of a subset of Zima's type.

DEFINITION 2. Let $E$ be a vector space over IR and \|\|$^{*}$ : $E \rightarrow[0, \infty)$ so that the following conditions are satisfied:

1. For every $x \in E,\left.|| x\right|^{*}=||-x \|^{*}$ and ||$x||^{*}<>0=x=0$.

2. For every $x, y \in E:|| x+y\left\|^{*} \leq|| x||^{*}+|| y\right\|^{*}$.

3. If $\left\|x_{n}-x_{0}\right\|^{*} \rightarrow 0\left(x_{n} \in E, n \in \mathbb{N} \cup\{0\}\right)$ and $t_{n} \in I R(n \in \mathbb{I N})$, $t_{n} \rightarrow t_{0}$, when $n \rightarrow \infty$, then $\left\|t_{n} x_{n}-t_{0} x_{0}\right\|^{*} \rightarrow 0, n \rightarrow \infty$.

Then \|\|$^{*}$ is said to be a paranorm and the pair $\left(E,\|\|^{*}\right)$ a paranormed space. 
If $\left(E,\|\|^{*}\right)$ is a paranormed space then $E$ is a topological vector space in which the fundamental system of neighbourhoods of zero is given by the family $V=\left\{V_{r}\right\}_{p>0}$, where:

$$
V_{r}=\left\{\left.x|||x|\right|^{*}<r\right\} \text {. }
$$

Let $K$ be a nonempty subset of $E$ where $\left(E,\|\|^{*}\right)$ is a paranormed space. The following condition is introduced in [16]. Suppose that there exists $C(K)>0$ so that:

(2) $\|t x\|^{*} \leq C(K) t \mid\|x\|^{*}$, for every $t \in[0,1]$ and every $x \in K-K$. It is easy to see that from (2) we obtain:

$$
\operatorname{co}\left(U_{\frac{r}{C(K)}} \cap(K-K)\right) \subseteq U_{r} \text {, for every } r>0 .
$$

Let $S(0,1)$ be the space of finite measurable functions (classes) on the interval $[0,1]$ with the metric $d(\hat{x}, \hat{y}), \hat{x}, \hat{y} \in S(0,1)$ defined by:

$$
d(\hat{x}, \hat{y})=\int_{0}^{1} \frac{|x(t)-y(t)|}{1+|x(t)-y(t)|} \mu(d t), \quad \begin{aligned}
& \{x(t)\} \in \hat{x} \\
& \{y(t)\} \in \hat{y} .
\end{aligned}
$$

The space $S(0,1)$ is also a paranormed space with the paranorm:

$$
\|x\|^{*}=\int_{0}^{1} \frac{|x(t)|}{1+|x(t)|} \mu(d t), \hat{x} \in S(0,1) \text {. }
$$

Let $M>0$ and $K_{M}$ be the subset of $S(0,1)$ defined by:

$$
\begin{gathered}
K_{M}=\{\hat{x}, \hat{x} \in S(0,1), \quad|x(t)| \leq M, t \in[0,1] \text { for some } \\
\{x(t)\} \in \hat{x}\} .
\end{gathered}
$$

We proved in [6] that $C\left(K_{M}\right)$ from (2) is in this case $1+2 M$.

\section{A Coincidence Theorem for Multivalued mappings.}

The following lema is a generalization of proposition 2 from Browder's paper [1].

LEMMA. Let $E$ and $F$ be topological vector spaces, $C$ a compact subset of $E, T: C \rightarrow 2_{c o}^{F}$ an upper semicontinuous mapping such that for a given convex subset $A$ of $F$ : 


$$
T(x) \cap A \neq \emptyset \text { for every } x \in C
$$

and $T(C)$ be of Zima's type. Let $V_{0}$ be a neighbourhood of zero in $F$ and $U_{0}$ a neighbourhood of zero in $E$. Then there exists a continuous singlevalued mopping $f: C \rightarrow \operatorname{co} M, M \subseteq A$, card $M<\infty$ such that for each $x \in C$, there exists $u \in C$ such that $x \in u+U_{o}$ and $f(x) \in T(u)+V_{0}$.

Proof. Let $\tilde{V}_{0}$ be a neigbourhood of zero in $F$ such that $\operatorname{co}\left(\tilde{V}_{0} \cap(T(C)-T(C))\right) \subseteq V_{0}$. Since the set $T(C)$ is of zima's type such a neighbourhood exists. Further, the mapping $T$ is upper semicontinuous and so there exists, for each $x \in C$, a neighbourhood $U_{x}$ of zero in $E$, such that:

$$
v \epsilon x+U_{x} \Rightarrow T(v) \subseteq T(x)+\tilde{V}_{0} .
$$

We shall suppose that $U_{x} \subseteq U_{0}$, for every $x \in C$. Let $V_{x}$ be an open symmetric neighbourhood of zero in $E$ such that $V_{x}+V_{x} \subseteq U_{x}$. From the compactness of the set $C$ it follows that there exists a finite open covering : $\left\{x_{j}+V_{x_{j}}\right\}_{j=1}^{m}$ of the set $C$.

Let $W=n_{j=1}^{m} V_{x_{j}}$ and $\left\{v_{1}, v_{2}, \ldots, v_{n}\right\} \subseteq C$ so that:

$$
C \subseteq \mathrm{u}_{s=1}^{n}\left\{v_{s}+W\right\}
$$

Let $\left\{h_{1}, h_{2}, \ldots, h_{n}\right\}$ be a partition of unity for the covering $\left\{v_{s}+W\right\}_{s=1}^{n}$ and $y_{s} \in T\left(v_{s}\right) \cap A \neq \emptyset$, for every $s \in\{1,2, \ldots, n\}$. As in Browder's paper [1], let us define the mapping $f$ in the following way:

$$
f(x)=\sum_{s=1}^{n} h_{s}(x) y_{s}, \text { for every } x \in C .
$$

It remains to be proved that the mapping $f$ satisfies all the conditions which are given in the Lemma. Suppose that $x \in C$ and let $j \in\{1,2, \ldots, m\}$ be such that $x \in x_{j}+V_{x_{j}}$. If $s \in\{1,2, \ldots, n\}$ is such that $h_{s}(x) \neq 0$ 
then $x \in v_{x}+W \subseteq v_{s}+V_{x_{j}}$. Since $V_{x_{j}}$ is symmetric we obtain $v_{s} \epsilon x+V_{x_{j}} \subseteq x_{j}+V_{x_{j}}+V_{x_{j}} \subseteq x_{j}+U_{x_{j}}$. Then (3) implies that $T\left(v_{s}\right) \subseteq$ $T\left(x_{j}\right)+\widetilde{V}_{0}$ and since $y_{s} \in T\left(v_{s}\right)(s \in\{1,2, \ldots, n\})$ we obtain:

$$
h_{s}(x) \neq 0 \Rightarrow y_{s} \in T\left(x_{j}\right)+\tilde{V}_{0} \text {. }
$$

Let us prove that $f(x) \in T\left(x_{j}\right)+V_{0}$. From $y_{s} \in T\left(x_{j}\right)+\tilde{V}_{0}$ it follows that there exists $z_{s} \in T\left(x_{j}\right)$ and $u_{s} \in \widetilde{v}_{0}$ such that $y_{s}=z_{s}+u_{s}$. Further, since $y_{s} \in T\left(v_{s}\right)$ and $z_{s} \in T\left(x_{j}\right)$ it follows that $u_{s} \in \widetilde{V}_{0} \cap(T(C)-T(C))$. From the definition of the mapping $f$ we obtain, for every $x \in C$ :

$$
\begin{aligned}
f(x)= & \sum_{s: h_{s}(x) \neq y_{s}}=\sum_{s: h_{s}(x) \neq 0} h_{s}(x)\left(z_{s}+u_{s}\right)= \\
= & \sum_{s: h_{s}(x) \neq 0} h_{s}(x) z_{s}+\sum h_{s}(x) u_{s} .
\end{aligned}
$$

Since $T\left(x_{j}\right)$ is convex, from the relation $z_{s} \in T\left(x_{j}\right)$, for every $s$ such that $h_{s}(x) \neq 0$, we obtain:

$$
\sum_{s: h_{s}(x) \neq 0}(x) z_{s} \in T\left(x_{j}\right) .
$$

On the other hand, from $u_{s} \in \tilde{V}_{0} \cap(T(C)-T(C)$ ), for every $s$ such that $h_{s}(x) \neq 0$, we obtain:

$$
\sum_{s: h_{s}(x) \neq 0} h_{s}(x) u_{s} \in \operatorname{co}\left(\tilde{V}_{o} \cap(T(C)-T(C))\right) .
$$

From (4) and (5) we have that:

$$
f(x) \in T\left(x_{j}\right)+\operatorname{co}\left(\tilde{V}_{o} n(T(C)-T(C))\right) \subseteq T\left(x_{j}\right)+V_{o}
$$

which implies for $u=x_{j}$ that the condition for $f$ in the Lemma is satisfied. 
Remark. Since every subset of a locally convex space is of Zima's type from the Lemma, Proposition 2 follows from [1].

THEOREM. Let $C$ be a compact, convex subset of a topological vector space $E, C_{1}$ a compact, convex subset of a topological vector space $F, T: C \rightarrow 2{ }_{C O}^{C_{1}}$ an upper semicontinuous mapping, $S: C_{1} \rightarrow 2_{\text {co }}^{C}$ an upper semicontinuous mopping such that the sets $T(C), S\left(C_{1}\right)$ are of zima's type. Then there exist $x_{0} \in C$ and $y_{0} \in T\left(x_{0}^{\prime}\right.$ so that $x_{0} \in S\left(y_{0}\right)$.

Proof. The proof is similar to the proof of Theorem 1, fxom [1]. By $G(T)$ and $G(S)$ we shall denote graphs of $T$ and $S$ respectively. Let $U$ be any neighbourhood of zero in $E$ and $V$ any neighbourhood of zero in $F$. Let us prove that:

$$
G(T) \cap\left(G\left(S^{-1}\right)+(U \times V)\right) \neq \emptyset .
$$

Let $\tilde{U}$ be a neighbourhood of zero in $E$ such that $\tilde{U}+\widetilde{U} \subseteq U$, and $\widetilde{V}$ a neighbourhood of zero in $E$ such that $\widetilde{V}+\widetilde{V} \subseteq V$. From the Lemma it follows that there exist a continuous mapping $f_{1}$ of $C$ into $C_{1}$ such that:

(i) For each $x \in C$ there exists $(u, v) \in G(T)$ such that $x \in u+\tilde{y}$ and $f_{1}(x) \in v+\tilde{V}, f_{1}(C) \subseteq \operatorname{Lin}(M), \operatorname{card} M<\infty\left(M \subseteq C_{1}\right)$ and a continuous mapping $f_{2}: C_{1} \rightarrow C$ such that:

(ii) For each $y \in C_{1}$, there exists $\left(v_{1}, u_{1}\right) \in G(S)$ such that $y \in v_{1}+\tilde{V}$ and $f_{2}(y) \in u_{1}+\tilde{U}, f_{2}\left(C_{1}\right) \subseteq \operatorname{Lin}(P), \operatorname{card} P<\infty(P \subseteq C)$. since $f_{2} f_{1}: C \rightarrow C$ is a continuous mapping such that $f_{2} f_{1}(C)$ is a subset of the linear hull of a finite subset of $C$, from Brouwer's fixed point theorem it follows that there exists $x_{0} \in C$ so that $f_{2} f_{1}\left(x_{0}\right)=x_{0}$.

Let $u, v, u_{1}$ and $v_{1}$ are chosen from (i) and (ii) for $x=x_{0}$ and $y=y_{0}=f_{1}\left(x_{0}\right)$. Then $x_{0} \in u+\tilde{U}$ and $x_{0}=f_{2}\left(y_{0}\right) \in u_{1}+\tilde{U}$ and if we suppose that $\widetilde{U}$ is symmetric we obtain $u-u_{1} \in \tilde{U}+\widetilde{U} \subseteq U$. similarly 
$v-v_{1} \epsilon \tilde{V}+\tilde{V} \subseteq V$, if we suppose that $\tilde{V}$ is symmetric. Hence $(u, v) \in\left(u_{1}, v_{1}\right)+U \times V$ which implies (6).

COROLLARY 1. Let $C=C_{1}, E=F$ and $S=I d_{C}$ in the Theorem. Then $T$ has a fixed point.

Remark. If in the Theorem we suppose, instead of the assumption that $S\left(C_{1}\right)$ is of Zima's type, that $S(\overline{C O T}(C))$ is of zima's type, it is obvious that the Theorem remains valid, since we can take $C_{1}^{\prime}=\overline{\cot }(C)$. If $F$ is complete and $C_{1}$ is of zima's type then from the Remark after Definition 1 it follows that it is enough to suppose that $C_{1}$ is closed and convex.

If $S=I d_{C}$ in [3] is proved a fixed point theorem if $T(C)$ is of Zima's type.

COROLLARY 2. Let $c$ be a compact convex subset of topological vector space $E, C_{1}$ a nonempty subset of topological vector space $F$, $T: C \rightarrow 2{ }_{c o}^{C_{1}}$ and $S: C_{1} \rightarrow 2_{\text {co }}^{C}$ an upper semicontinuous mapping such that $\overline{c o} S(T(C))$ is of Zima's type. If for every $x \in C$ there exists $y \in C_{1}$ such that $x \in \operatorname{intT}^{-1}(y)$ then there exists $x_{0} \in C$ and $y_{0} \in T\left(x_{0}\right)$ such that $x_{0} \in S\left(y_{0}\right)$.

Proof. As in [14] it follows that there exists a continuous mapping $f: C \rightarrow C_{1}$ such that $f(x) \in T(x)$, for every $x \in C$. For the sake of completeness we include the proof. Since $C$ is compact, from $c=u_{y \in C_{1}} \operatorname{in} T^{-1}(y)$, it follows that there exists a finite set $\left\{y_{1}, y_{2}, \ldots\right.$, $\left.y_{n}\right\}$ fxom $C_{1}$ such that $c=u_{i=1}^{n} T^{-1}\left(y_{i}\right)$. Let $\left\{f_{1}, f_{2}, \ldots, f_{n}\right\}$ be a partition of unity subordinated to the covering $\left\{\text { int } T^{-1}\left(y_{i}\right)\right\}_{i=1}^{n}$ and let $f: C \rightarrow F$ be defined in the following way: 


$$
f(x)=\sum_{i=1}^{n} f_{i}(x) y_{i}, x \in C
$$

If $f_{i}(x) \neq 0$ then $x \in \operatorname{int} T^{-1}\left(y_{i}\right) \subseteq T^{-1}\left(y_{i}\right)$ and so we have the implication: $f_{i}(x) \neq 0 \Rightarrow y_{i} \in T(x)$.

Hence, $f$ is a continuous mapping from $C$ into $C_{1}$ such that $f(x) \in T(x)$, for every $x \in C$, and let $R(x)=S(f(x))$, for every $x \in C$. Then $R$ is an upper semicontinuous mapping from $C$ into $2_{c o}^{C}$ and $R(C) \subseteq S(T(C))$ which implies that $\overline{c o R}(C)$ is of zima's type. From this it follows that there exists $x_{0} \in C$ such that $x_{0} \in R\left(x_{0}\right)=$ $S\left(f\left(x_{0}\right)\right)$. If we take that $y_{0}=f\left(x_{0}\right)$ the proof is complete.

As an application, we can prove a Proposition about the existence of a maximal element in the sense given below [14].

DEFINITION 3. Let $X$ be a subset of a topological vector space $E$ and for every $x \in X, T x$ is a subset of $X$ (may be empty). A point $x_{0} \in X$ is said to be a maximal element of $T$ if $T\left(x_{0}\right)=\emptyset$.

PROPOSITION. Let $c$ be a compact convex subset of a topological vector space $E, C_{1}$ a nonempty convex subset of a topological vector space $F$, for every $x \in C, T x \subseteq C_{1}$ and $S$ an upper semicontinuous mapping of $C_{1}$ into ${ }_{2}^{C}$ such that $T x \neq \varnothing$ implies: $x \in S(c o T x)$ and there exists $y_{x} \in C_{1}$ such that $x \in \operatorname{int} T^{-1}\left(y_{x}\right)$. If $\overline{\cos }\left(C_{1}\right)$ is of Zima's type there exists $x_{0} \in C$ such that $T x_{0}=\emptyset$. Proof. Suppose that $T x \neq \emptyset$, for every $x \in C$. Then co $T x \neq \emptyset$ and the mappings $S$ and $G: x \rightarrow$ co $T x$ satisfy all the conditions of Corollary 2. From this we obtain that there exists $x_{0} \in C$ such that $x_{0} \in S\left(G\left(x_{0}\right)\right)=S\left(\operatorname{coT} x_{0}\right)$ which is a contradiction. So there exists $x_{0} \in C$ such that $T x_{0}=\emptyset$. 


\section{References}

[1] F. E. Browder, "Coincidence theorems, minimax theorems, and variational inequalities", Contemp. Math. 26(1984), 67-80.

[2] Büi Cong Cuōng, "Some Fixed Point Theorems for Miltifunctions in Topological Vector Spaces (announcement of results)." Buzz. Polish Acad. Sci. Math., 32,3-4 (1984), 215-221.

[3] 0 . Haďzic, "Some fixed point and almost fixed point theorems for multivalued mappings in topological vector spaces", Nonlinear Anal., 5,9(1981), 1009-1019.

[4] O. Hadžić, "On Kakutani's Fixed Point Theorem in Topological Vector Spaces", Bulz. Polish Acad. Sci., 30. 3-4(1982), 141-144.

[5] o. Hadžic, "On equilibrium point in topological vector spaces", Comment Math. Univ. Carolin., 23, 4(1982), 727-738.

[6] O. Hadžić, "On Sadovski's fixed point theorem in topological vector spaces", Comment Math.Prace Mat. 24(1984), 51-55.

[7] o. Hadžić, Fixed Point Theory in Topological Vector Spaces, Institute of Mathematics, Novi Sad, 1984, $337 \mathrm{pp}$.

[8] O. Hadžić, "A theorem on the fixed point for multivalued mappings in topological vector spaces", Rend. Istit. Mat. Univ. Trieste (to appear).

[9] O. Hadžić, Lj. Gajic, "A fixed point theorem for multivalued mappings in topological vector spaces", Fund. Matin., 109(1980), 163-167.

[10] o. Hadžić, Lj. Gajic, "Some Applications of Fixed Point Theorems for Multivalued Mappings on Minimax Problems in Topological Vector Spaces", Math. Operationsforsch. Statist. Ser. Optim., 15 (1984), 193-201.

[1] S. Hahn, "Zur Leray-Schauder-Theorie in topologischen Vektorraümen", Wiss. 2. Tech. Univ. Dresden 24 (1975), 375-378.

[12] s. Hahn, "Über die stabilitat von Lösungen nichlinearer Operatorengleichungen in nicht notwending lokalkonvexen topologischen vektorraumen", Comment. Math. Univ. Carolin. 17, 3 (1976), 421-440. 
[13] S. Hahn, T. Riedrich, "Der Abbildungsgrad kompakter Vektorfelder in nicht notwendig lokalkonvexen topologischen vektorräumen", Wiss. Z. Tech. Univ. Dresden, 22 (1973), 37-42.

[14] E. Tarafdar and G. Mehta, "The existence of quasi-equilibrium in a competitive economy", Internat. J. Sci. Eng. 1, 1 (1984), 1-12.

[15] N. Yannelis and N. Prabhakar, "Existence of maximal elements and equilibria in linear topological spaces", J. Math. Econom. 12 (1983), 233-245.

[16] K. Zima, "On the Schauder fixed point theorem with respect to paranormed spaces", Comment. Math. Prac. Math., 19 (1977), 421-423.

University of Novi Sad

Faculty of Science

Department of Mathematics

Dr Ilije Duričiča 4

21000 NOVI SAD

YUGOSIIAVIA 